\title{
HeartCare+: A Smart Heart Care Mobile Application for Framingham-Based Early Risk Prediction of Hard Coronary Heart Diseases in Middle East
}

\author{
Hoda Ahmed Galal Elsayed, ${ }^{1}$ Mariam Ahmed Galal, ${ }^{2}$ and Liyakathunisa Syed ${ }^{3}$ \\ ${ }^{1}$ Software Engineering, Prince Sultan University, Riyadh, Saudi Arabia \\ ${ }^{2}$ Alfaisal University, Riyadh, Saudi Arabia \\ ${ }^{3}$ CCIS, ACM, Prince Sultan University, Riyadh, Saudi Arabia
}

Correspondence should be addressed to Hoda Ahmed Galal Elsayed; helsayed1993@gmail.com

Received 14 March 2017; Accepted 5 July 2017; Published 25 September 2017

Academic Editor: Jesus Fontecha

Copyright (C) 2017 Hoda Ahmed Galal Elsayed et al. This is an open access article distributed under the Creative Commons Attribution License, which permits unrestricted use, distribution, and reproduction in any medium, provided the original work is properly cited.

\begin{abstract}
Background. Healthcare is a challenging, yet so demanding sector that developing countries are paying more attention to recently. Statistics show that rural areas are expected to develop a high rate of heart diseases, which is a leading cause of sudden mortality, in the future. Thus, providing solutions that can assist rural people in detecting the cardiac risks early will be vital for uncovering and even preventing the long-term complications of cardiac diseases. Methodology. Mobile technology can be effectively utilized to limit the cardiac diseases' prevalence in rural Middle East. This paper proposes a smart mobile solution for early risk detection of hard coronary heart diseases that uses the Framingham scoring model. Results. Smart HeartCare+ mobile app estimates accurately coronary heart diseases' risk over 10 years based on clinical and nonclinical data and classifies the patient risk to low, moderate, or high. HeartCare+ also directs the patients to further treatment recommendations. Conclusion. This work attempts to investigate the effectiveness of the mobile technology in the early risk detection of coronary heart diseases. HeartCare+ app intensifies the communication channel between the lab workers and patients residing in rural areas and cardiologists and specialist residing in urban places.
\end{abstract}

\section{Introduction}

Middle East is experiencing a wide growth of population nowadays with a noticeable decrease of medical and manpower resources which makes it vulnerable to hard coronary heart diseases (HCHD) attacks. These resources are represented by staff, expert physicians, and expensive medical equipment. Moreover, life style changes have always been the reason chasing the HCHD prevalence in urban cities but a recent scary fact reflected that rural areas' records of HCHD mortality were found higher compared to the urban areas $[1,2]$. HCHD's prevalence rate in rural areas was $38.8 \%$ higher than urban areas [3-8]. Rural areas suffer from poor clinical services, limited facilities, and equipment purchases, full time medical staff shortage, and lack of funding. Accordingly, people living in such areas do not receive enough medical care as they are being margined in most of the countries. In addition, heart diseases management in rural areas is being challenged by different risk factors that participate in the cardiac events (e.g., diabetes, obesity, smoking, and poverty) $[2,9]$. Furthermore, underdeveloped regions of rural areas suffer from low ratio of doctors available to treat patients in multiple towns nearby which may violate the number of patients per doctor rule set by medical standards. Adding to that, the financial cost of visiting doctors in the urban cities can be unbearable burden for rural people beside the treatment expenses. Lack of medical care awareness among rural population made the process of managing HCHD more challenging [10]. This sharp increase in HCHD prevalence motivated many researchers lately to investigate the possibility of controlling such chronic diseases in the Middle Eastern countries. 
In our attempt to provide a solution for HCHD' early prediction, we found that the current indicators suggest an increase in the smart phone usage rate by 2020 to be around 6.1 billion worldwide [11]. In addition, Middle East is recording the highest percentage of Internet users so far who are smart phone users simultaneously [12]. Moreover, advances in mobile technology as well as its being in hands along with its low cost and their popularity make it an ideal platform for developing healthcare solutions for early detection of HCHD [13]. Accordingly, mobile-based solutions can potentially support the early detection of possible $\mathrm{HCHD}$ risks early, which will thus help patients in rural areas avoid having a chronic heart disease in the future by simple lifestyle changes. This research aims to early detect HCHD risks for rural patients over 10 years using Framingham scoring (FRS) model [14]. Choosing FRS, in particular, was due to its outperformance to predict HCHD in comparison with other models [15] beside its ability to be generalized from one population to another [16]. In the FRS scoring model's risk estimation depends on collecting sample data from each patient. The risk percentage is then evaluated and recommendations are given based on the risk class.

This type of smart healthcare solution has not yet been deployed in the Middle East. Furthermore, no healthcare solution is designed to connect the HCHD patients with their doctors for risk detection and prevention. This research contributes by its role to the 2030 vision introduced by many countries in the Middle East (http://vision2030.gov.sa/en, http://sdsegypt2030.com/?lang=en, http://www.qdb.qa/English/Investing/Pages/Qatar2030.aspx) which supports

(1) preventive care given to different genders and ages to reduce chronic diseases (e.g., heart diseases),

(2) providing home care when necessary,

(3) reducing visits to specialists and consultants,

(4) extending the population reach by medical services,

(5) improving the service's quality introduced by the small healthcare units,

(6) establishing advanced and integrated health systems,

(7) bearing the costs of healthcare visits and treatments.

This study aims to assess patients HCHD risks severity through the proposed mobile-based solution to avoid the possible long-term consequences. This paper proposes a smart healthcare solution that not only predicts the HCHDs' risks early but also enables patients, lab workers, and cardiologists to communicate. In other words, the proposed solution enables rural patients to receive their clinical data from the lab workers remotely and send their risk results to cardiologists of their choice for medical recommendations. We suggest that such solution will have its positive effect in reducing the communication gaps between rural patients, lab workers, and urban physicians. Adding more to that, it can positively mitigate the high costs of big clinics visits and over budget treatments.

This research is organized as follows: Section 1 presents introduction to CHD prevalence in the rural areas, Section 2 presents the related work, and in Section 3 the proposed methodology adopted in the design and implementation is presented, whereas Sections 4 and 5 present the proposed mobile app framework and its requirements, respectively. The results of mobile application implementation are presented in Section 6, and Section 7 presents the conclusion to our work on this research and our findings.

\section{Related Work}

A study done by Singh et al. [13] introduced CVDMagic, a mobile-based solution that detects CVD in small Indian clinics and spreads awareness about newly emerging diseases. They proposed a new approach that consumes less time compared to the traditional paper based process. The CVDMagic was designed for clinics' workers to use through their mobile phones to calculate the risk of certain patient. Their approach relies on patient's face to face meetings with the clinic workers to know the possible risk on their hearts. They introduced a system that focused on females with middle to old age because they are more prone to CVD risks.

Another study by Jin et al. [18] shed the light on the importance of using mobile phones in detecting and preventing CVD through ECG signals classification. The authors proposed HeartToGo, a mobile phone that is capable of tracking and recording the ECG signals in real time using wearable sensors and accelerometer. The system then generates a heart summary report based on the extracted signal features. However, their system is considered costly as it requires other sensors and devices beside the mobile phone. Moreover, it does not allow for communication with a doctor, and it suggests that patients will be able to fully understand the generated report on their own as well.

University of West Indies has proposed MediNet [19, 20], a mobile-based health management system under development, that is designed to help patients to get the necessary cure for diabetes and CVD using telephonic intervention. It targets the regions with poor healthcare facilities, but a strong cellular phone infrastructure. The system moves the data, collected from the patient monitoring devices, to a central server where data about severe cases are sent to the medical officers. ClickMedix is a mobile social enterprise by MIT (http://web.mit.edu/) to connect patients suffering from diabetes with physicians quickly. DoctorFromHome (https://doctorfromhome.in/) is an online service designed for both doctors and patients. Patients with common diseases (like colds, fevers, stomach pain, and weight-loss problems) can easily get advices to recover faster using their portable devices.

Another study by Ramachandran et al. [21] demonstrated the effectiveness of mobile devices' usage in the medical services to reduce the maternal health dangers during pregnancy. The authors proposed a prototype written in Java 2 Micro Edition (J2ME) to spread awareness towards pregnancy risks through text messages and enhance the communication between the patient and the rural healthcare worker simultaneously. The proposed prototype supports the presentation of messages through audio or video to 
help the lab workers who are not so well trained and thus are not credible to convince the patients. In their study, they reported that the phone-based dialogue messages can effectively improve the quality of counseling sessions.

A study done by Gluckman in 2016 discussed another tool named ASCVD risk estimator that was released by the American Heart Association (AHA) to measure the risk value of CVD over 10 years. ASCVD risk estimator was created in two forms: mobile application and web-based application for easy access through browsers in various portable devices. In the process of risk calculation, the application requires the patient to enter the following data: age, gender, race (white/black), diabetic and smoking status as well as HDL, blood pressure, and hypertension treatment. Moreover, the application can be accessible for educative purposes by both patients and clinicians for recommendations and general medical nutrition information.

The primary purpose of the previous studies was to design solutions that can assist the healthcare workers to estimate the CVD risk using traditional way of data collection. Moreover, the focus was on CVD not HCHD, CVD was their main target, and thus risk scoring models (like Framingham) were not used. Furthermore, some studies focused on applying machine learning techniques on CVD's risk prediction process not $\mathrm{HCHD}$. As observed, systems that were designed to be applicable in rural healthcare units did not allow the patients to communicate with their physicians, for further recommendations, or with the clinic workers, for clinical data collection. Moreover, most of the introduced solutions are released in English language which may not be applicable in rural Middle Eastern countries. A summary of our observations on these studies is shown in Table 1 comparison of our proposed solution "HeartCare+" with other related works.

This study aims to ease the communication between the following entities: (1) patients in rural areas, (2) cardiologists in urban areas, and (3) rural area's healthcare workers (lab workers). In addition, it focuses on providing a mobile application that uses a machine learning technique from data collection to automated HCHD's risk and survival prediction. Through the proposed mobile application, the patient can receive the clinical data required for risk estimation from lab workers remotely and send the risk report to cardiologists for recommendations.

Although phone calls and text messages are good means for communication, the solution proposed in this research will allow patients to communicate with cardiologists located anywhere in any part of the world from the rural area. This gives the option for patients to choose among expert doctors with high level of experience to follow up their cases. Moreover, they can get the recommendations without having to pay for additional clinical visits which might be costly beside treatment expenses. The proposed solution requires the clinics workers to provide only the clinical data per each patient where the patients can take care of nonclinical data necessary for risk estimation. Furthermore, it will also reduce the demands on the clinics side if the patients with HCHD risks are to be tracked remotely.

\section{Proposed Methodology}

3.1. Framingham's CHD Risk Factors. Framingham heart study suggests that different HCHD biomarkers can effectively estimate the risk over 10 years. These biomarkers include age, gender, total cholesterol, HDL, SBP, hypertension treatment, smoking status, diabetic status, and family history of HCHD. The relation between these risk factors in risk estimation is illustrated in Figure 1. The categorical data with dichotomous values are given as gender, smoking status, family history, and diabetic status, shown in Figure 1.

3.2. Risk Calculation Using Logistic Regression Model. In this section, the mathematical model for calculating the 10-year risk of the chosen Framingham model is illustrated from (1) to (6b). This method is based on a mathematical model that uses logistic regression models [22] in replacement to the original discriminant models $[23,24]$. The following calculations are based on Cox hazard regression model [25-29]. A general formula is used to estimate the risk over specific duration as per the model used but then the model is tailored according to the gender [30-33].

\subsubsection{Cox Time-to-Event Survival Model for HCHD Risk Prediction}

$S(t \mid r)$

$=[\text { Average survival in population }]^{\exp (\text { beta of each risk factor) }}$,

where

(i) the average survival varies based on the subject gender;

(ii) the beta of each risk factor is a linear function;

(iii) $S(t \mid r)$ represents the survival of a risk event by time (subjects with risk factors $=$ mean of risk factors)

$S(t \mid r)$

$=[\text { Average survival in population }]^{\exp \left(\sum_{i=1}^{n}\left(\beta_{i}\left(R_{i}-M_{i}\right)\right)\right)}$,

where

(i) $R_{i}$ is a vector of $R_{1}, \ldots, R_{n}$ which represents the relative risk factors for specific subject;

(ii) $\beta_{i}$ is a vector of $\beta_{1}, \ldots, \beta_{n}$ which represents the logs of relative risk factors;

(iii) $M_{i}$ is a vector of $M_{1}, \ldots, M_{n}$ which represents the means of the risk factors in certain population

$S(t r)$

$=[\text { Average survival in population }]^{\exp \left(\sum_{i=1}^{n}\left(\beta_{i} R_{i}-\beta_{i} M_{i}\right)\right)}$

$\simeq[\text { Average survival in population }]^{\exp \left(\sum(\beta R-\beta \text { Mean } R)\right)}$. 


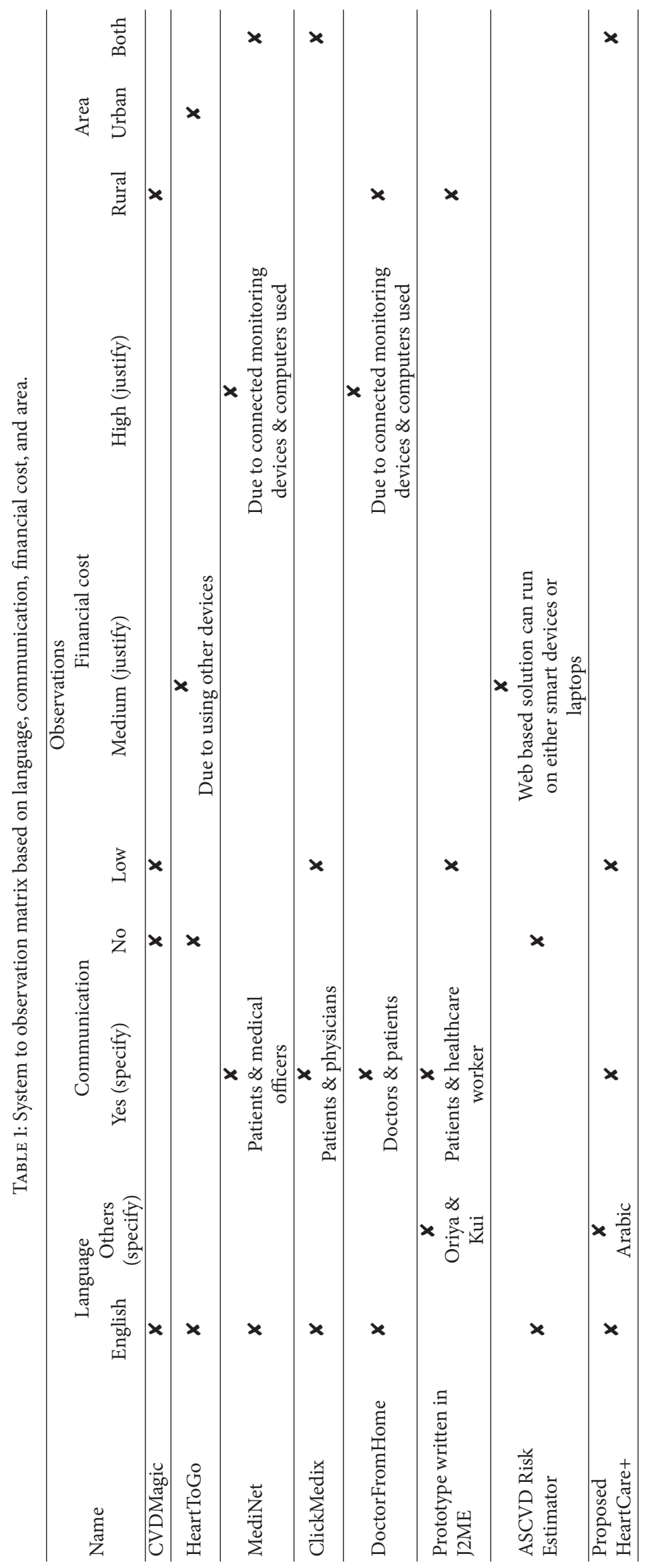




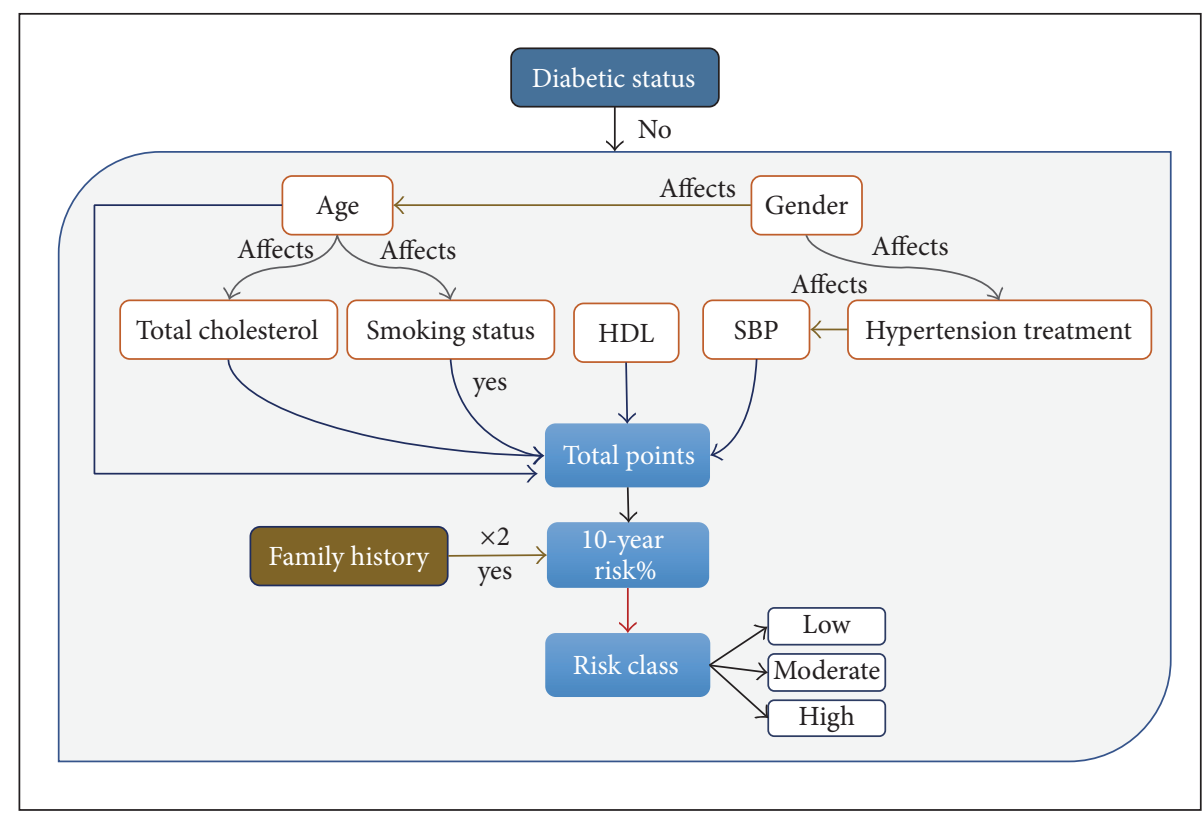

FIGURE 1: Framingham's HCHD risk factors [17].

Applying formula expansion,

$S(t r)$

$=[\text { Average survival in population }]^{\exp \left(\left(\beta_{1}\left(R_{1}-M_{1}\right)\right)+\cdots+\left(\beta_{n}\left(R_{n}-M_{n}\right)\right)\right)}$.

\section{General Formula}

$P(t \mid r)=[\log (T)$

- Average survival in population $]^{\exp \left(\left(\beta_{1}\left(R_{1}-M_{1}\right)\right)+\cdots+\left(\beta_{n}\left(R_{n}-M_{n}\right)\right)\right)}$,

where

(i) $T$ is the number of years to estimate the HCHD risk which is 10 in the chosen Framingham model;

(ii) $P(t \mid r)$ is the probability of time to risk survival;

(iii) $\log (T)=\log (10)=1$

$P(t r)=[1$

- Average survival in population $]^{\exp \left(\left(\beta_{1}\left(R_{1}-M_{1}\right)\right)+\cdots+\left(\beta_{n}\left(R_{n}-M_{n}\right)\right)\right)}$

Substituting the average survival for men,

10-years risk (men)

$$
=[1-0.940200]^{\exp \left(\left(\beta_{1}\left(R_{1}-M_{1}\right)\right)+\cdots+\left(\beta_{n}\left(R_{n}-M_{n}\right)\right)\right)} .
$$

Substituting the average survival for women,

10-years risk (women)

$$
=[1-0.987670]^{\exp \left(\left(\beta_{1}\left(R_{1}-M_{1}\right)\right)+\cdots+\left(\beta_{n}\left(R_{n}-M_{n}\right)\right)\right)} .
$$

Both 0.940200 and 0.987670 values, the average survival for men and women, respectively, are identified by the Framingham heart study (FHS) (https://www.framinghamheartstudy.org/risk-functions/coronary-heart-disease/hard-10year-risk.php) in 2017 based on the Adult Treatment Panel III JAMA in 2001.
3.3. Risk Estimation Stages. According to the Framingham risk functions [33], the chosen 10-year HCHD model has five stages, shown in Figure 2, to assess the severity of the risk class which are (1) checking if patient has no diabetes, (2) calculating the total points and mapping the total points to risk percentage, based on (1) to (6b), for both genders, (3) mapping the total points to risk percentage, (4) double checking the risk\% based on the CHD family history, and (5) defining the risk class based on a given criterion [14, 34]. If patient has no diabetes, the model is applicable to be used; otherwise, the patient should use another appropriate model. Total risk points are calculated by summing the values assigned to the risk factors based on patient's data. The summed total of these points is then mapped to risk percentage. Risk percentage is recalculated based on the family history. If the CHD family history is set to true, the previous risk $\%$ is multiplied by 2 ; otherwise, it should remain the same. The risk class is then defined based on the following criteria: (1) low risk class if risk\% is less than 10, (2) moderate risk class if the risk\% is between 10 and 19 inclusive, and (3) high risk class if the risk\% is equal to or more than $20[14,34]$. Based on these risk categories, treatment recommendations are made [35].

As an Example, Given the Patient's Data as Follows

(i) Normal glucose level (no diabetes)

(ii) Age is " 75 "

(iii) Gender is "male"

(iv) Smoking status is set to "true"

(v) HDL is " $14.58 \mathrm{mg} / \mathrm{dl}$ ”

(vi) Total cholesterol is " $93.6 \mathrm{mg} / \mathrm{dl}$ "

(vii) SBP is " $140 "$ 


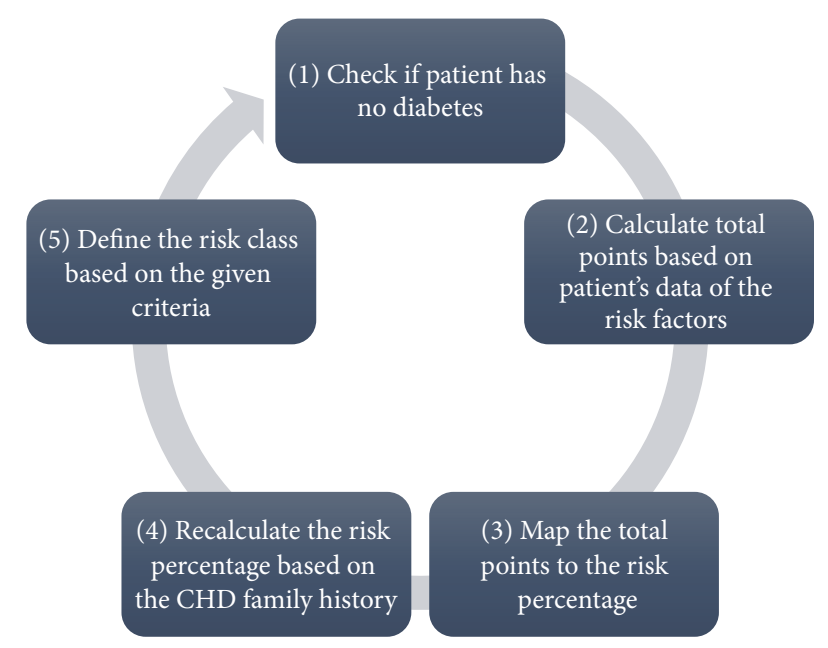

Figure 2: Stages of calculating 10-year risk using Framingham model.

(viii) Hypertension status is set to "true"

(ix) CHD family history is set to "true"

The Process of Assigning a Risk Class Label Based on the Four Stages Is Illustrated as Follows

Stage 1. Check if patient has no diabetes.

Normal glucose level (no diabetes) means that the model is suitable for predicting the patient's CHD risk.

Stage 2. Calculate the total points based on (1) to (6b).

(i) Age is “ 75 ", male $\rightarrow 13$ points

(ii) Smoking status is set to "true" $\rightarrow 1$ points

(iii) HDL is " $14.58 \mathrm{mg} / \mathrm{dl}$ " $\rightarrow 2$ points

(iv) Total cholesterol is " $93.6 \mathrm{mg} / \mathrm{dl}$ " $\rightarrow 0$ points

(v) Treated hypertension, SBP is " 140 " $\rightarrow 2$ points

Total points $=13+1+2+0+2=18$ points.

Stage 3. Map the total points to risk percentage: 15 points represent $6 \%$ risk.

Stage 4. Double check the risk\% based on the CHD family history.

CHD family history is set to "true" which means that the risk $\%=6 \% * 2=12 \%$.

Stage 5. Define the risk class based on a given criterion.

$12 \%$ is between 10 and 19 which indicates that the patient's risk is moderate.

In this study, exclusion criterion is set to dismiss all data that does not fit in the Framingham scoring model. This exclusion will help in reducing the level of inaccuracy of the proposed mobile application. This exclusion criterion includes dismissing patients' data with age below 20 or greater than 79 .

\section{Proposed Mobile Application Framework}

The suggested model is divided into rural side and urban side shown in Figure 3. The rural side involves two user categories which are the patient and lab workers of the small healthcare units in rural Middle Eastern countries. On the other urban side, expert cardiologists with high experience level represent the only user category. In Figure 3, numerical labels are scattered to show the workflow of the mobile application activities and the communication process among involved users. These labels describe the following action steps.

Step 1. The patient can choose a cardiologist to follow up his/her case through the mobile application.

Step 2. Once the doctor confirms supervising the patients, healthcare's lab worker will be able to add the nonclinical data to the patient's file based on the previous patient visit while the patient is supposed to fill only the nonclinical data. Clinical data includes diabetic status that depends on the glucose level, HDL, total cholesterol, hypertension treatment, and SBP. Nonclinical data represent simple questions that can be easily answered by the patient (e.g., age, gender, family history to $\mathrm{CHD}$, and smoking status). The reason for this data division is to reduce the amount of error that may result if the patients had to fill the nonclinical data on their own without being familiar with some medical terminologies.

Step 3. Both clinical and nonclinical data are combined.

Step 4. The risk class will be estimated to be low, moderate, or high based on the combined data.

Step 5. Report will be generated which included all risk factors' inputs and predicted risk class.

Step 6. The report will be sent to the cardiologist in charge of monitoring the patient case.

Step 7. The cardiologist can send back the recommendation to the patient based on the generated report.

The framework in Figure 3 suggests that all involved user will communicate through the proposed HeartCare+ mobile application. The mobile application uses the biomarkers of the 10-year HCHD risk estimation model based on the Framingham equations (1) to (6b). The model calculates and estimates whether the patient is at low, moderate, or high risk based on the entered data.

In the sections to follow, the requirements of the mobile application are illustrated. Some of these requirements have been elicited from the literature review as well as similar systems.

\section{Mobile Application Requirements}

This mobile application should reflect the medical requirements of the Framingham scoring model to estimate the risk over 10 years. A summary of the mobile application 


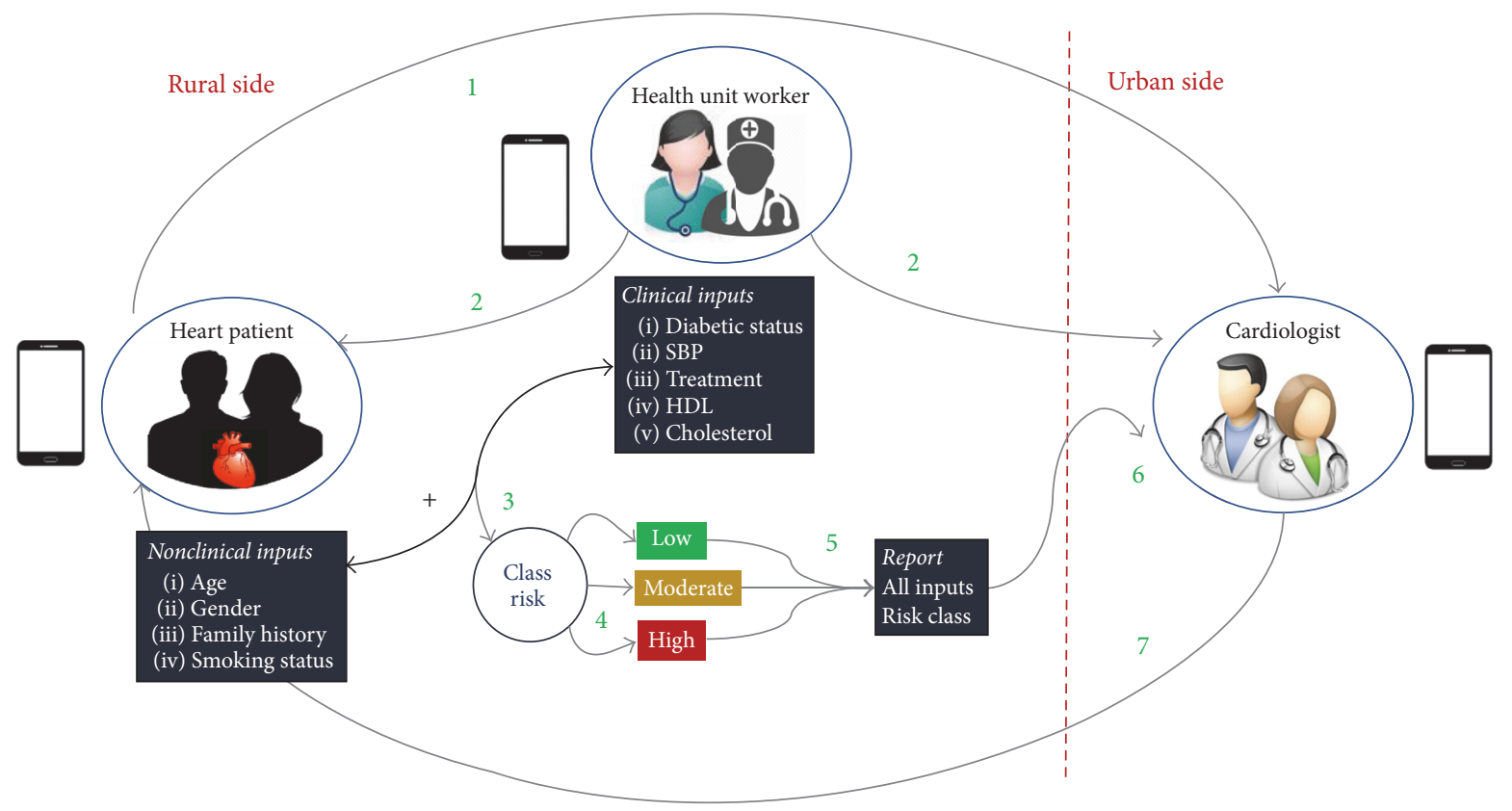

FIgURE 3: Suggested framework for the mobile application.

requirements is shown in Figure 4 . Figure 4 shows the usecase diagram of the mobile application requirements. The main actors involved in the HCHD-FRED mobile application are (1) patients, (2) lab workers, (3) cardiologists, and (4) mobile application. The patient can be either low risk patient, moderate risk patient, or high risk patient. The patient use cases are choosing cardiologist, filling the nonclinical data, and sending report to the cardiologist. The lab worker use cases are filling clinical data to patient's file. The cardiologist use cases are choosing the patients to follow their cases and sending the recommendation back after viewing their medical reports. Mobile app actor is responsible for estimating the possible risk over 10 years as well as generating a case report after filling the required data.

\section{Results and Discussion}

The proposed mobile-based solution estimates the HCHD risk based on clinical and nonclinical indicators based on the Framingham scoring model. HeartCare+ app involves the following user categories: patients, lab workers, and cardiologists. The whole process starts when the patient logs in to the proposed system shown in Figure 5(a). The patient interface will then appear with two actions in the sliding panel which are doctors list and ongoing checks shown in Figure 5(b). The patient can choose a doctor from the list to follow up his/her case shown in Figure 5(c). In the ongoing checks screen, the patient request to the doctor chosen will appear in a pending status shown in Figure 5(d). If the patient clicked on the pending request, a partial report with only the nonclinical data, filled by the patient during the registration, will appear.

Secondly, the cardiologist must confirm the patient's request in order for the patient file to be available to the lab worker, who fills the clinical data of that certain patient. In Figure 6, this process takes place when the cardiologist logs in to HeartCare+ app shown in Figure 6(a). The cardiologist interface will then appear where the available actions are shown in the sliding panels which are available checklists and ready lab reports shown in Figure 6(b). The list will then appear with all the patients who sent the request to that cardiologist where the cardiologist can confirm supervising any of these patients by clicking on the patient name shown in Figure 6(c).

Thirdly, the lab worker will get access to the patient file after the cardiologist's confirmation. This starts when the lab worker logs in to HeartCare+ app shown in Figure 7(a). The lab worker will then have access to the patient file when he/she clicks on the check-options of the sliding panel shown in Figures 7(b) and 7(c). When the lab worker clicks on the patient file, he/she will be able to fill the clinical data related to that patient and they can send these data afterwards to both the patient and assigned cardiologist shown in Figure $7(\mathrm{~d})$.

Accordingly, both the patient and the cardiologist will be able to view the full report with the assigned risk class based on the clinical and nonclinical data of the patient. The patient check results will appear in the checks list as completed shown in Figure 8(a). Similarly, the cardiologist can find a new lab report on his/her interface with the patient name and medical record number (MRN) shown in Figure 8(b). Once the patient or the cardiologist clicks on the newly added check result that appears on the list, a full report with the risk estimation will be shown in Figure 8(c). Afterwards, both patient and cardiologist can exchange messages for further inquiries or recommendations shown in Figure 8(d). Furthermore, this interface with all its steps is also supporting the Arabic which makes HeartCare+ application more reliable and applicable solution in the Middle East as well as in the Western world. 


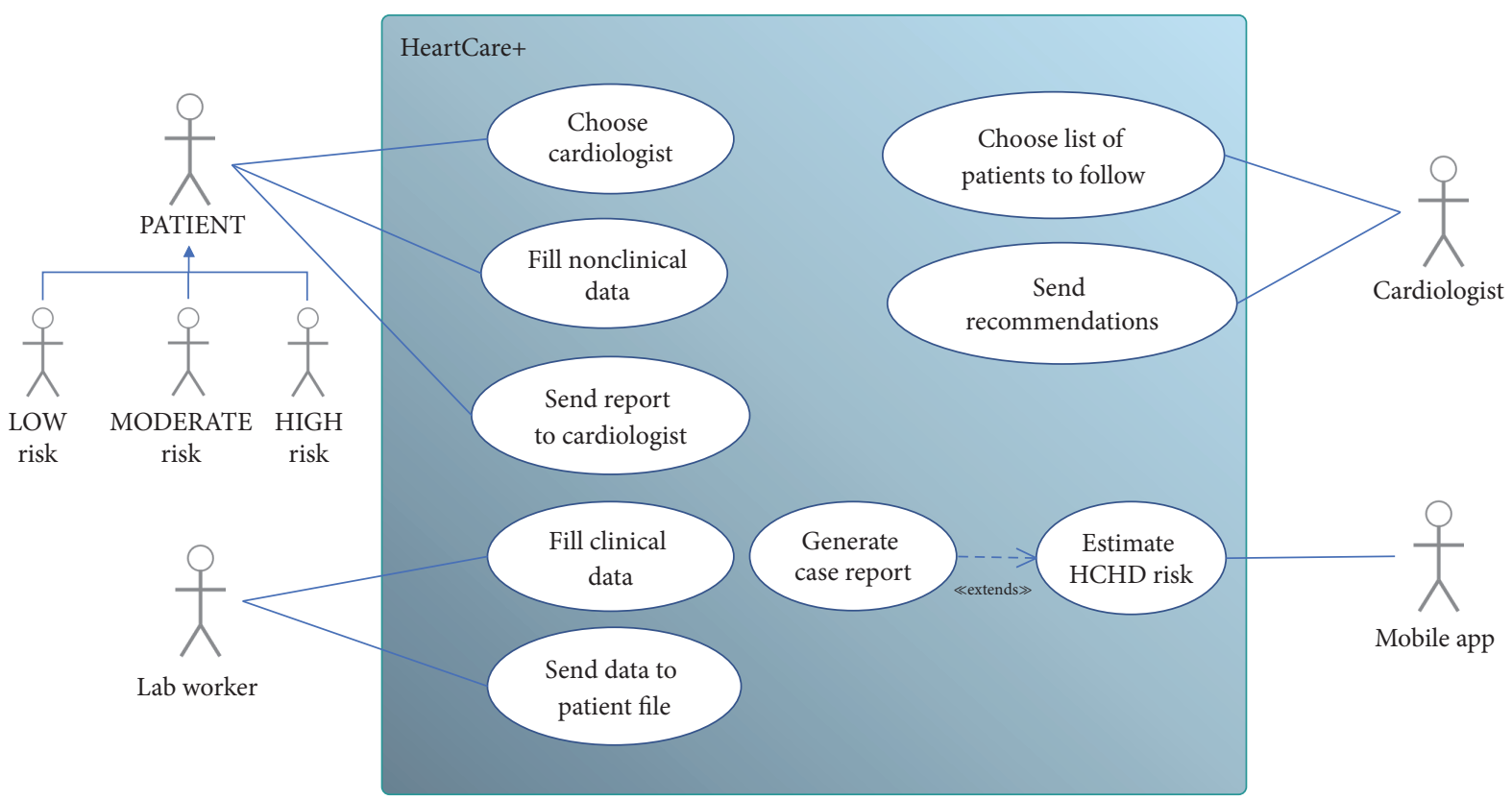

FIGURE 4: Use-case diagram of proposed application.

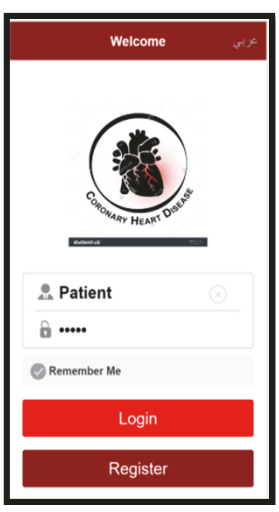

(a) Patient logs in

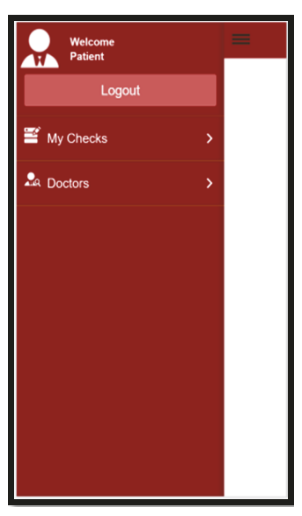

(b) Patient's sliding actions panel

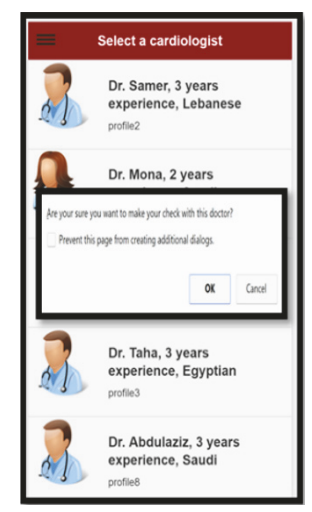

(c) Patient chooses a doctor from the list

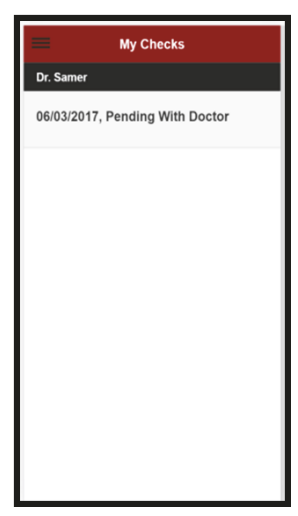

(d) Pending check awaits doctor's confirmation

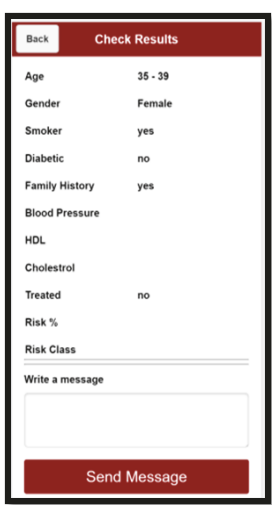

(e) Partially filled patient report

FIgUre 5: Patient logs in to the HeartCare+ app to choose his/her cardiologist.

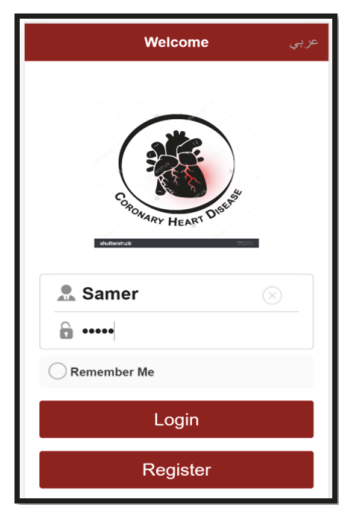

(a) Cardiologist logs in

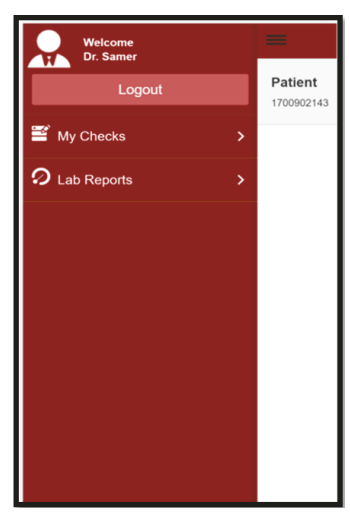

(b) Cardiologist's sliding actions panel

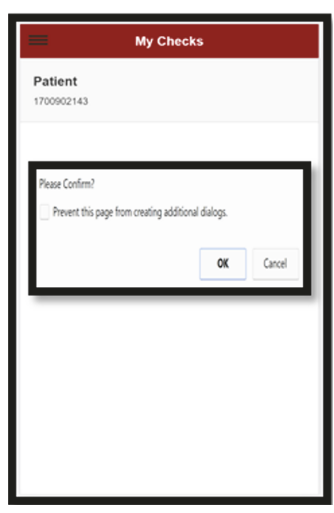

(c) Cardiologist confirms the pending request

FIgURE 6: Cardiologist confirms the patient request on HeartCare+ app. 


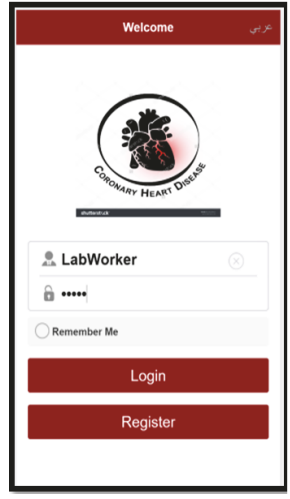

(a) Lab worker logs in

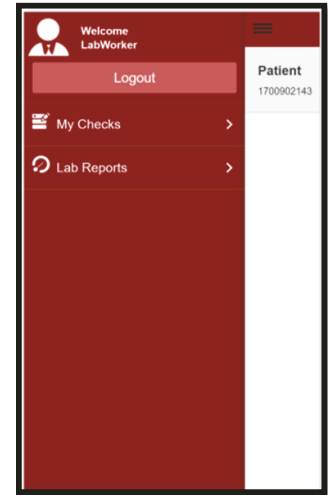

(b) Lab worker's sliding actions panel

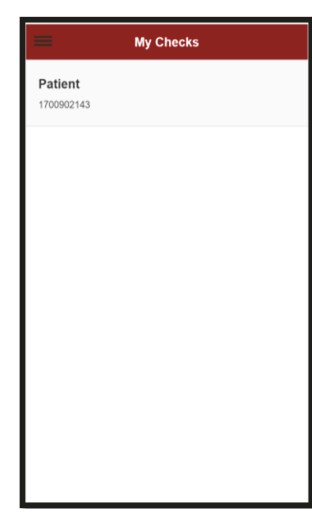

(c) Awaiting patient file list

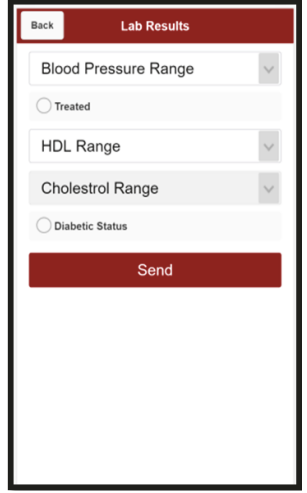

(d) Lab worker fills the clinical data

Figure 7: Lab worker will have access to the patient file to fill the clinical data on HeartCare+ app.

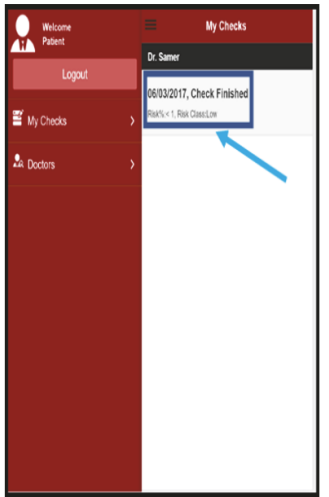

(a) Patient check status changed with a brief result summary

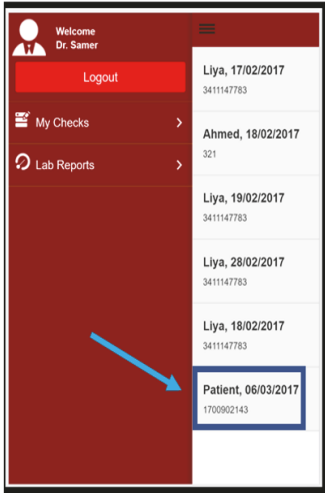

(b) A new lab report with patient name and MRN on doctor's interface

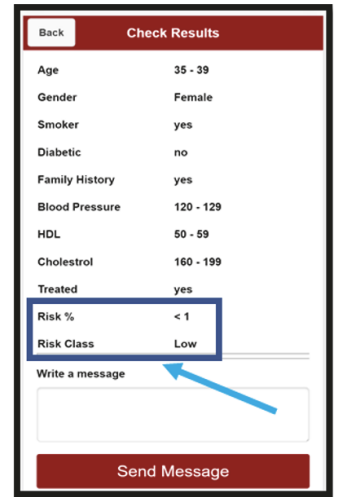

(c) Complete lab report that appears to both patient and cardiologist

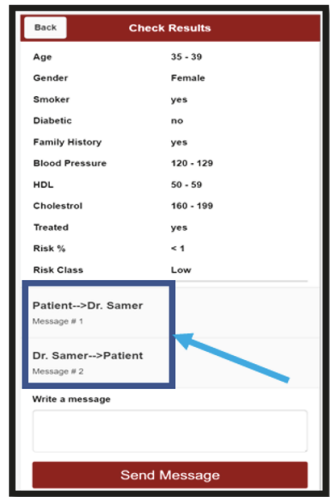

(d) Message exchange between patient and doctor for recommendations or extra inquiries

FIGURE 8: Both patient and cardiologist can view the complete risk report and exchange messages.

\section{Conclusion}

Growing risk of coronary heart diseases and lack of resources necessitate introducing a robust, yet low cost, solution for risk detection of Middle Eastern population in both rural and urban areas. In this work, we have proposed HeartCare+ mobile-based solution that is capable of accurately estimating the HCHD risk over 10 years based on medical measurements introduced by the Framingham heart study. Moreover, HeartCare+ app intensifies the communication channel between the lab workers and patients residing in rural towns and cardiologists in urban places. The application can classify the patient based on the clinical and nonclinical data to low, moderate, or high risk based on the Framingham scoring model's criteria. HeartCare+ app, however, has its own limitations that will be resolved in the future work. These limitations include the following: (1) application does not guarantee the identity of doctors registered on it but registering new doctors can be controllable by the Ministry of Health in the future and (2) all lab workers get any patient's file automatically which can be adjusted based on the clinic location in which the patient had his laboratory checks completed. Hence our proposed smart mobile helps in early risks prediction of coronary heart disease risks both in rural and urban areas.

\section{Conflicts of Interest}

The authors declare that there are no conflicts of interest regarding the publication of this paper.

\section{Acknowledgments}

The authors would like to extend their thanks to Dr. Liyakathunisa Syed and acknowledge Prince Sultan University for the continuous support and motivation to get this work done. Also, they would like to thank Dr. Mariam Ahmed Galal for the medical guidance involved in the mobile application. 


\section{References}

[1] A. Kulshreshtha, A. Goyal, K. Dabhadkar, E. Veledar, and V. Vaccarino, "Urban-rural differences in coronary heart disease mortality in the United States: 1999-2009," Public Health Reports, vol. 129, no. 1, pp. 19-29, 2014.

[2] A. Kulshreshtha, A. Goyal, K. Dabhadkar, E. Veledar, and V. Vaccarino, "Urban-rural differences in coronary heart disease mortality in the United States: 1999-2009," Public Health Reports, vol. 129, no. 1, pp. 19-29, 2014, Washington, D.C., 1974.

[3] A. O'Connor and G. Wellenius, "Rural-urban disparities in the prevalence of diabetes and coronary heart disease," Public Health, vol. 126, no. 10, pp. 813-820, 2012.

[4] V. Mohan, R. Deepa, and S. S. Rani, "Prevalence of coronary artery disease and its relationship to lipids in a selected population in South India," Journal of the American College of Cardiology, vol. 38, pp. 682-687, 2001.

[5] R. Gupta, V. P. Gupta, M. Sarna et al., "Prevalence of coronary heart disease and risk factors in an urban Indian population: Jaipur heart watch-2,' Indian Heart Journal, vol. 54, no. 1, pp. 59-66, 2002.

[6] M. A. Kamili, I. H. Dar, G. Ali, H. S. Wazir, and S. Hussain, "Prevalence of coronary heart disease in Kashmiris," Indian Heart Journal, vol. 61, pp. 44-49, 2007.

[7] A. K. Gupta, A. Bharadwaj, and S. Ashotra, "Feasibility and training of multipurpose workers in detection, prevention and control of coronary artery disease in apple-belt of Shimla hills," South Asian Journal of Preventive Cardiology, vol. 6, pp. 17-22, 2002.

[8] R. Kumar, M. C. Singh, and S. K. Ahlawat, "Urbanization and coronary heart disease: a study of urban-rural differences in northern India," Indian Heart Journal, vol. 58, pp. 126-130, 2006.

[9] W. Almahmeed, M. Samir Arnaout, R. Chettaoui et al., "Coronary artery disease in africa and the middle east," Therapeutics and Clinical Risk Management, vol. 8, pp. 65-72, 2012.

[10] A. Singh, V. Naik, S. Lal et al., "Improving the efficiency of healthcare delivery system in underdeveloped rural areas," in Proceedings of the 2011 3rd International Conference on Communication Systems and Networks COMSNETS '11, January 2011.

[11] I. L. (June 2015). 6.1B Smartphone Users Globally By 2020, Overtaking Basic Fixed Phone Subscriptions, https://techcrunch .com/2015/06/02/6-1b-smartphone-users-globally-by-2020overtaking-basic-fixed-phone-subscriptions/.

[12] J. Poushter, "Smartphone Ownership and Internet Usage Continues to Climb in Emerging Economies," Pew Research Center, Washington, wash, USA, 1-5, 2016, http://www.pewglobal.org/2016/02/22/smartphone-ownership-and-internetusage-continues-to-climb-in-emerging-economies/.

[13] P. Singh, A. Singh, V. Naik, and S. Lal, "CVDMagic, a mobile based study for CVD risk detection in rural India," in Proceedings of the Fifth International Conference on Information and Communication Technologies and Development, pp. 359-366, 2012.

[14] H. C. Kim, "Clinical utility of novel biomarkers in the prediction of coronary heart disease," Korean Circulation Journal, vol. 42, no. 4, pp. 223-228, 2012.

[15] M. O. Versteylen, I. A. Joosen, L. J. Shaw, J. Narula, and L. Hofstra, "Comparison of Framingham, PROCAM, SCORE, and Diamond Forrester to predict coronary atherosclerosis and cardiovascular events," Journal of Nuclear Cardiology, vol. 18, no. 5, pp. 904-911, 2011.
[16] S. Aghtar, "A new incremental classification approach monitoring: the risk of heart disease," 2012, https://macsphere .mcmaster.ca/bitstream/11375/12637/1/fulltext.pdf.

[17] H. Elsayed and L. Syed, "An Automatic Early Risk Classification of Hard Coronary Heart Diseases using Framingham Scoring Model," in Proceedings of the 2nd International Conference on Internet of Things, Data and Cloud Computing (ICC '17), 2017.

[18] Z. J. Z. Jin, J. Oresko, S. H. S. Huang, and C. Cheng, "HeartToGo, A personalized medicine technology for cardiovascular disease prevention and detection," in Proceedings of the Life Science Systems and Applications Workshop, pp. 80-83, 2009.

[19] P. Mohan and S. Sultan, "MediNet: a mobile healthcare management system for the Caribbean region," in Proceedings of the 2009 6th Annual International Conference on Mobile and Ubiquitous Systems: Networking and Services, MobiQuitous '09, July 2009.

[20] P. Mohan, D. Marin, S. Sultan, and A. Deen, "MediNet: personalizing the self-care process for patients with diabetes and cardiovascular disease using mobile telephony," in Proceedings of the 30th Annual International Conference of the IEEE Engineering in Medicine and Biology Society, pp. 755-758, Vancouver, Canada, August 2008.

[21] D. Ramachandran, V. Goswami, and J. Canny, "Research and reality: Using mobile messages to promote maternal health in rural India," in Proceedings of the 4th ACM/IEEE International Conference on Information and Communication Technologies and Development, ICTD '10, December 2010.

[22] S. H. Walker and D. B. Duncan, "Estimation of the probability of an event as a function of several independent variables," Biometrika, vol. 54, no. 1, pp. 167-179, 1967.

[23] J. Truett, J. Cornfield, and W. Kannel, "A multivariate analysis of the risk of coronary heart disease in Framingham," Journal of Chronic Diseases, vol. 20, no. 7, pp. 11-24, 1967.

[24] J. Cornfield, T. Gordon, and W. Smith, "Quantal response curves for experimentally uncontrolled variables," Bulletin of the International Statistical Institute, vol. 28, 1961.

[25] P. W. F. Wilson, R. B. D’Agostino, D. Levy, A. M. Belanger, H. Silbershatz, and W. B. Kannel, "Prediction of coronary heart disease using risk factor categories," Circulation, vol. 97, pp. 1837-1847, 1998.

[26] R. B. D’Agostino Sr., S. Grundy, L. M. Sullivan, P. Wilson, and Group CHDRP, "Validation of the Framingham coronary heart disease prediction scores: results of a multiple ethnic groups investigation," The Journal of the American Medical Association, vol. 286, no. 2, pp. 180-187, 2001.

[27] P. A. Wolf, R. B. D’Agostino, A. J. Belanger, and W. B. Kannel, "Probability of stroke: a risk profile from the framingham study," Stroke, vol. 22, no. 3, pp. 312-318, 1991.

[28] R. B. D’Agostino, P. A. Wolf, A. J. Belanger, and W. B. Kannel, "Stroke risk profile: adjustment for antihypertensive medication the framingham study," Stroke, vol. 25, no. 1, pp. 40-43, 1994.

[29] R. B. D’Agostino Sr., R. S. Vasan, M. J. Pencina et al., "General cardiovascular risk profile for use in primary care: the Framingham heart study," Circulation, vol. 117, no. 6, pp. 743-753, 2008.

[30] R. B. DAgostino, M. J. Pencina, J. M. Massaro, and S. Coady, "Cardiovascular disease risk assessment: insights from framingham," Global Heart, vol. 8, no. 1, pp. 11-23, 2013.

[31] J. R. Lewis, W. Lim, S. S. Dhaliwal et al., "Estimated glomerular filtration rate as an independent predictor of atherosclerotic vascular disease in older women," BMC Nephrology, vol. 13, p. $58,2012$. 
[32] K. M. Anderson, P. W. F. Wilson, P. M. Odell, and W. B. Kannel, "An updated coronary risk profile. A statement for health professionals," Circulation, vol. 83, no. 1, pp. 356-362, 1991.

[33] Risk Score Profiles Framingham Heart Study, http://www.framinghamheartstudy.org/risk/index.html.

[34] M. O. Versteylen, I. A. Joosen, L. J. Shaw, J. Narula, and L. Hofstra, "Comparison of Framingham, PROCAM, SCORE, and Diamond Forrester to predict coronary atherosclerosis and cardiovascular events," Journal of Nuclear Cardiology, vol. 18, no. 5, pp. 904-911, 2011.

[35] S. M. Grundy, "Primary prevention of coronary heart disease: Integrating risk assessment with intervention," Circulation, vol. 100, no. 9, pp. 988-998, 1999. 

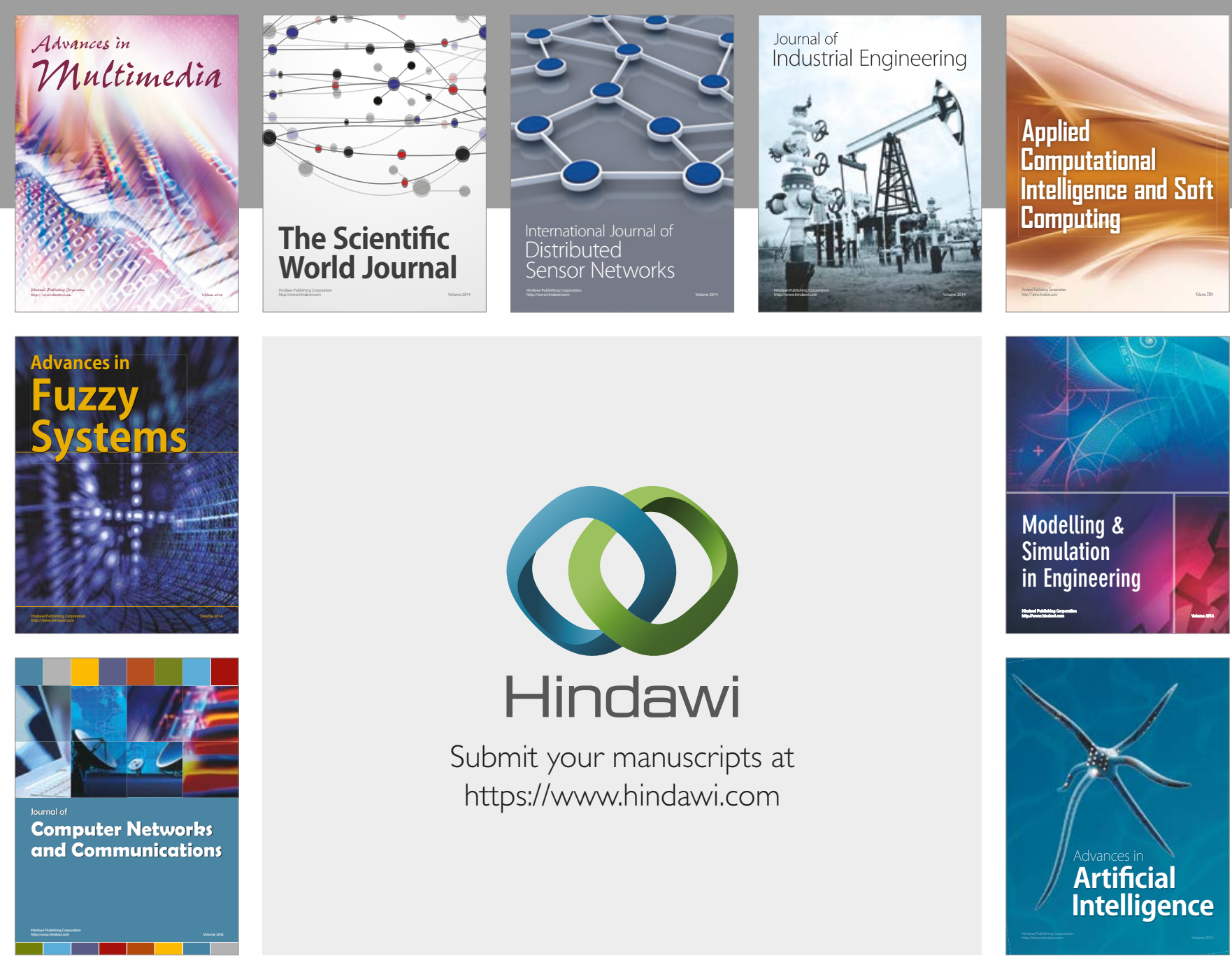

\section{Hindawi}

Submit your manuscripts at

https://www.hindawi.com
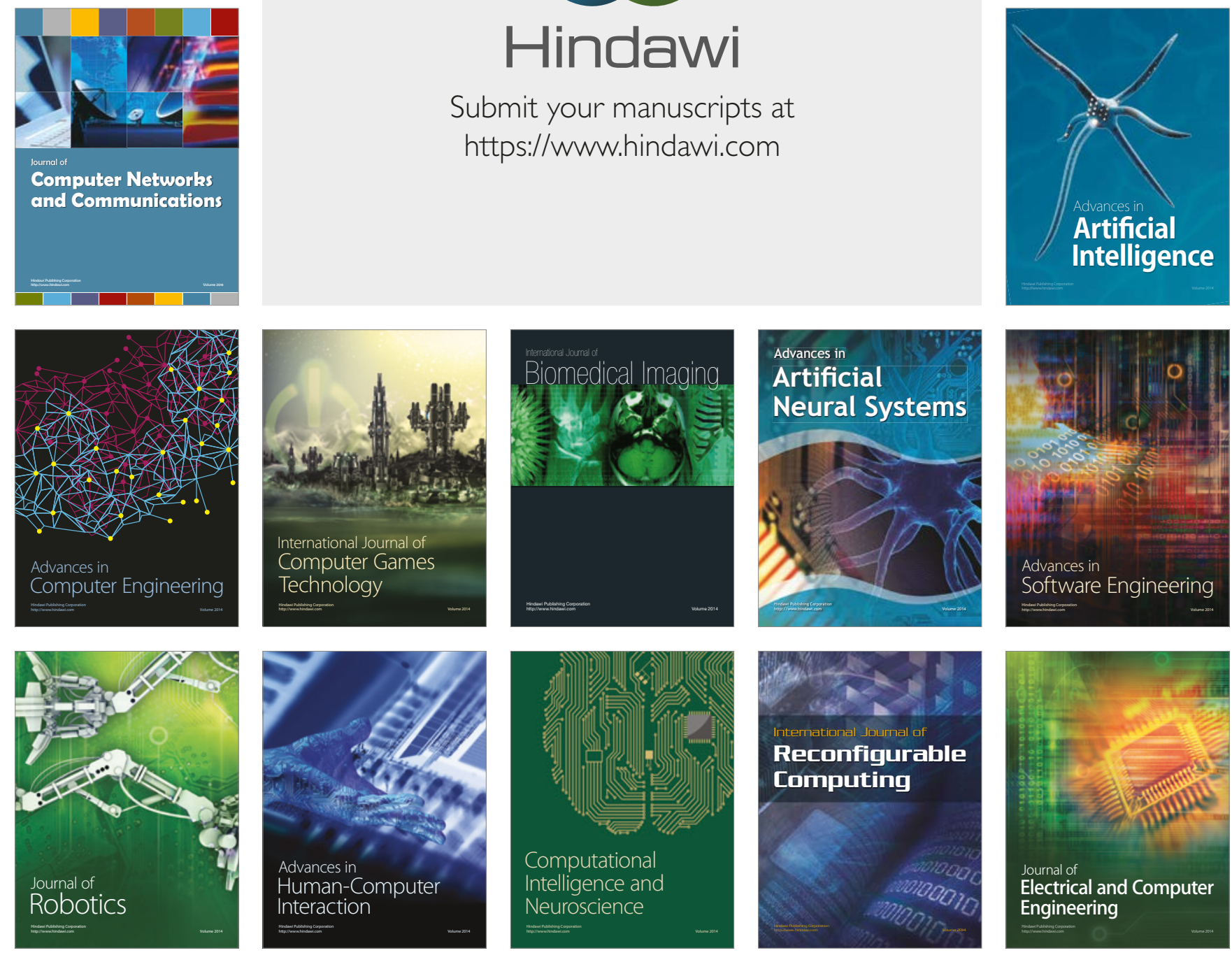